Original Article

Journal of Epilepsy Research pISSN 2233-6249 / eISSN 2233-6257

Received March 13, 2019

Revised June 2, 2019

Accepted June 24, 2019

Corresponding author:

Devendra Mishra, MD

Department of Pediatrics, Maulana Azad Medical College, 2, Bahadur Shah Zafar

Marg, Delhi 110002, India

Tel. +91-11-23236031

Fax. +91-11-23235574

E-mail; drdmishra@gmail.com

\title{
Clinico-Etiological Profile of Pediatric Refractory Status Epilepticus at a Public Hospital in India
}

\author{
KC Sadik, MD, Devendra Mishra, MD, Monica Juneja, MD, Urmila Jhamb, MD \\ Department of Pediatrics, Maulana Azad Medical College, Delhi, India
}

Background and Purpose: Refractory status epilepticus (RSE) has been infrequently studied in Indian children. This research was conducted to study the clinico-etiological profiles and short-term outcomes of children aged 1 month to 12 years with convulsive RSE, at a public hospital.

Methods: The study was conducted between 1st April 2016 and 28th February 2017 after receiving clearance from an Institutional Ethics Committee. All children (aged 1 month to 12 years) who presented to the pediatrics department of a tertiary-care public hospital with convulsive status epilepticus (SE), or who developed SE during their hospital stay, were enrolled. All patients were investigated and managed according to a standard protocol. Outcomes were assessed based on the Glasgow Outcome Scale. Details of children who progressed to RSE were compared to those without RSE.

Results: Fifty children (28 males) with CSE were enrolled, of which $20(40 \%)$ progressed to RSE. Central nervous system (CNS) infection was the most common etiology ( $53 \%$ in SE and $55 \%$ in RSE, $p>0.05$ ). Non-compliance with anti-epileptic drugs was the second most common etiology. The overall mortality rate was $38 \%$, and although the odds of death in RSE $(50 \%)$ were higher than in SE $(30 \%)$, this difference was not statistically significant $(p=0.15)$. The odds of having a poor outcome was six times higher in children with RSE as compared to those with SE (odds ratio, 6.0; 95\% confidence interval, 1.6-22.3; $p=0.005)$.

Conclusions: When managing CNS infections, pediatricians need to be aware of the high risk of developing RSE. In addition, the possibility of RSE should be considered and managed promptly in an intensive-care setting, to reduce the mortality and morbidity of this severe neurological condition. (2019;9:36-41)

Key words: Central nervous system infections, Complications, Drug therapy, Epilepsy, Mortality

\section{Introduction}

Status epilepticus (SE) is a life-threatening neurological emergency that requires immediate medical intervention, and is associated with high mortality and morbidity. ${ }^{1,2}$ The annual incidence of SE in children is reported to be $10-40$ per $100,000 .^{3}$ Refractory status epilepticus (RSE) is a more severe variant of SE, and was previously defined based on duration (i.e., when SE persisted for more than 1 or 2 hours). ${ }^{4}$ Presently, the most widely-accepted definition considers RSE to occur when seizures persist despite the administration of two appropriate anticonvulsants at acceptable doses, ${ }^{4}$ and is estimated to occur in around $10-40 \%$ of patients with SE. ${ }^{4,5}$ RSE has been shown to be associated with higher mortality and more neurological sequelae. ${ }^{6}$
Pediatric data from western countries on refractory SE using the new definition are available. ${ }^{7}$ On the other hand, the studies on RSE from this region are few, and they are primarily in adults. ${ }^{8,9}$ In addition, no recent systematic reviews on pediatric RSE are available from India, although Indian studies of pediatric SE include some RSE patients. ${ }^{10}$ Thus, this study was conducted to study the clinico-etiological profile of children with refractory convulsive SE, and to compare their profiles and outcomes to those of children with SE.

\section{Methods}

This longitudinal observational study was conducted in the pediatrics department of a large public hospital in New Delhi, India, over an 11-month period (1st April 2016 to 28th February 2017), after

This is an Open Access article distributed under the terms of the Creative Commons Attribution Non-Commercial License (http://creativecommons.org/licenses/by-nc/3.0/) which permits unrestricted non-commercial use, distribution, and reproduction in any medium, provided the original work is properly cited. 
Institutional Ethics Committee clearance. Informed written consent was obtained from the parents of all participants. All children (aged 1 month to 12 years) who presented with a convulsive $S E$, or those who developed SE during the hospital stay, were enrolled prospectively (Fig. 1).

SE was defined as a seizure lasting longer than 30 minutes, or recurrent seizures that lasted more than 30 minutes, during which the patient did not regain consciousness. ${ }^{11}$ RSE was defined as seizures that persisted despite the administration of two appropriate anticonvulsants at acceptable doses. ${ }^{4,5}$ Outcomes were assessed based on the Glasgow Outcome Scale (GOS), ${ }^{12}$ and were applied when the patient left the hospital, whether discharged or left against medical advice (LAMA). A poor outcome was defined as death, persistent vegetative state, or severe disability (GOS, categories 1-3).

After initial management and stabilization, a detailed history was obtained from parents, which included seizure type, seizure duration, treatment given outside hospital, any precipitating factors, etc. In subjects with previously-diagnosed epilepsy, details of their previous diagnosis and management were noted from records, making a special effort to determine any history of non-compliance and/or changes in drug dosage. Any previous imaging results, electroencephalography (EEG) results, and antiepileptic drug (AED) levels were also documented. Relevant developmental history and significant past history were documented. A detailed systemic examination, as far as possible, was carried out in all children.

A provisional diagnosis based on the patient's history and examination was made. A central nervous system (CNS) infection was defined as fever and seizures, along with features suggestive of meningitis, encephalitis or meningoencephalitis based on the hematological work-up, cerebrospinal fluid (CSF) analysis, blood/CSF culture, and neuroimaging. Febrile status was labelled when SE occurred in children (aged $<5$ years) who had been previously diagnosed with febrile seizures. ${ }^{13}$

Children were managed according to the standard management guidelines. ${ }^{5,14}$ Briefly, this consisted of two doses of intravenous midazolam followed sequentially by intravenous phenytoin, valproate and levetiracetam. Additionally, intravenous phenobarbitone bolus, midazolam infusion, or ketamine were used in the intensive care unit (ICU). Airway, breathing, and circulation were maintained. Once seizures were controlled, neuroimaging and EEG were per-

Children enrolled 50

46 from casualty

4 from inpatient wards

Given two intravenous bolus of midazolam, followed

by intravenous phenytoin (33)/valproate (17)
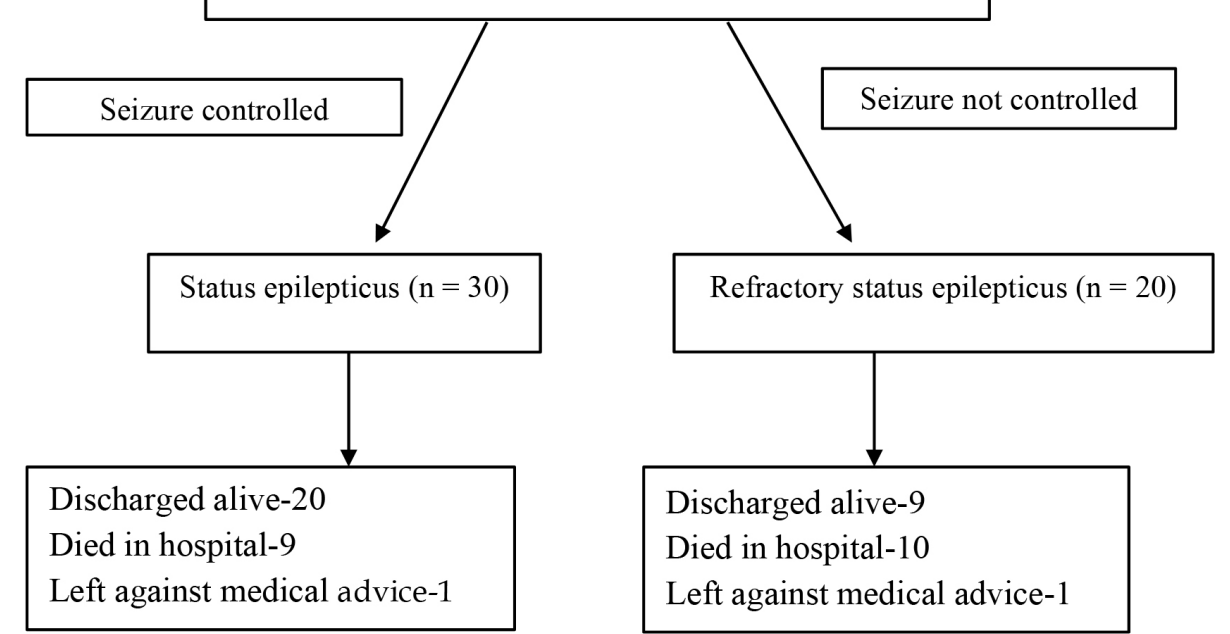

Figure 1. Study flow-chart. 
formed, and any additional required investigations were carried out. If the child was febrile, lumbar puncture was performed and CSF was collected for cytological examination, sugar-protein levels, and culturing. In patients in shock, for whom sampling studies were not possible initially, an initial diagnosis was made based on history and examination. Relevant samples were sent for analysis as soon as possible.

A detailed, structured form was filled out for each child, containing all of the above information. Children were monitored on a daily basis, with daily examinations and investigations performed as necessary. If beds were available in the pediatric ICU, children were shifted to the ICU. Otherwise, they were managed in the pediatric ward. Etiology and outcome were assessed in each child at the time of discharge/LAMA. The last examination's details were used to determine the GOS score, in case of patients absconding from the hospital.

\section{Statistical analyses}

A convenience sampling size of 50 SE patients was decided a priori. All data were compiled in an excel spreadsheet and were analyzed using Epi Info software. Various clinico-etiological characteristics and outcomes were compared between children with SE and children with RSE. We carried out univariate analyses to determine the odds of RSE with respect to clinical characteristics at admission,

A

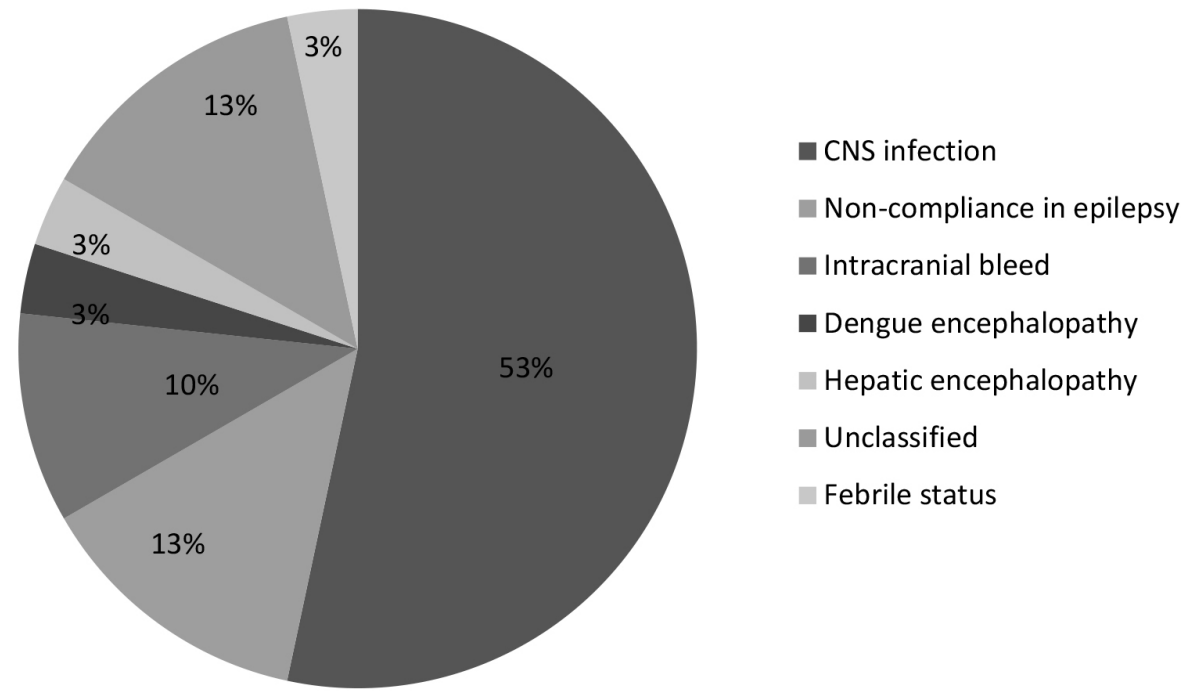

B

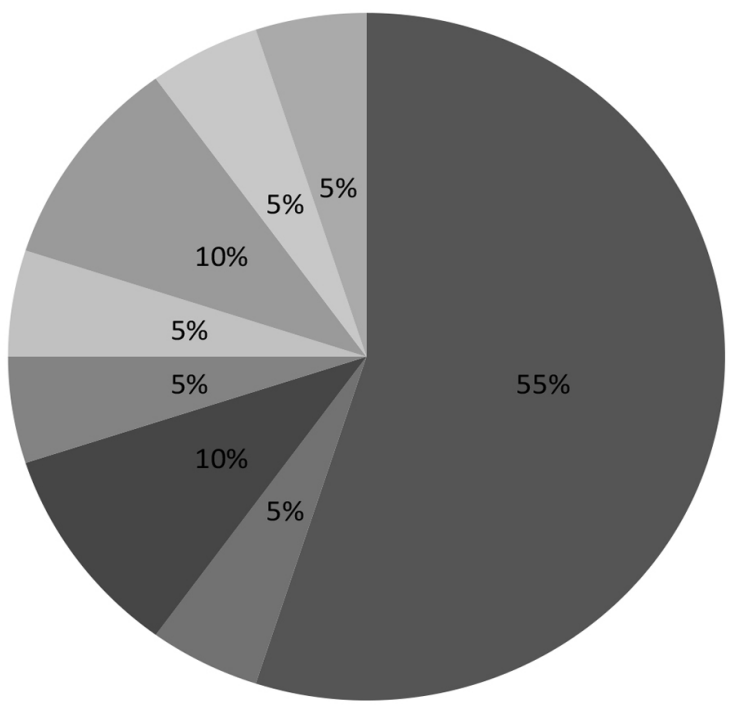

- CNS infection

- Metabolic

- Febrile status

Non-compliance of drugs in epiler

Intracranial bleed

- Unclassified

- Hepatic encephalopathy

- Cerebral atrophy

Figure 2. Pie chart showing the etiology in children with (A) status epilepticus $(n=30)$ and children progressing to (B) refractory status epilepticus ( $n=20)$. CNS, central nervous system. 
abnormal neuroimaging, or abnormal EEG. Risk factors for a poor outcome were explored. A $p$ value less than 0.05 was considered statistically significant.

\section{Results}

A total of 50 children (28 males) with SE were enrolled, of which 20 (40\%) progressed to RSE. The median (interquartile range) age was 3.5 years (3 months to 12 years). Two children in each group showed seizure onset after being admitted to the hospital, while the rest presented to the emergency department during a seizure. The median (range) distance from the patient residence to hospital was 7 (1-15) and 5 (1-15) km for the children in the two groups.

CNS infection was the most common etiology (53\% in SE and $55 \%$ in RSE; Supplementary Table 1), and non-compliance with anti-epileptic drugs was the next commonest etiology, with no difference between the groups (Fig. 2). The proportion of patients with pre-existing epilepsy, the duration of epilepsy prior to SE, and the epilepsy etiology (idiopathic/symptomatic) were not significantly different between the two groups. The majority of patients $(92 \%)$ had generalized seizures.

The risk of developing RSE was not significantly different between febrile children (odds ratio [OR], 1.17; 95\% confidence interval [CI], 0.34-3.9; $p=0.8$ ), children with epilepsy (OR, $0.69 ; 95 \% \mathrm{Cl}$, $0.18-2.7 ; p=0.6)$, children with developmental delays (OR, 1.25; $95 \% \mathrm{Cl}, 0.29-5.4 ; p=0.8)$, partial seizures (OR, $5.12 ; 95 \% \mathrm{Cl}$, $0.49-53.2 ; p=0.17)$, microcephaly (OR, $1.62 ; 95 \% \mathrm{Cl}, 0.35-7.4$; $p=0.5)$, shock at admission ( $\mathrm{OR}, 0.67 ; 95 \% \mathrm{Cl}, 0.19-2.36 ; p=0.5)$, or presence of a co-morbidity (OR, 1.76; $95 \% \mathrm{Cl}, 0.51-6.2 ; p=0.4$ ) (Table 1).

Nineteen (38\%) children died during the study, with CNS infection being the most common cause of death (84.2\%) (Table 2). The odds of death in children with RSE (50\%) were higher than those with SE (30\%), but the difference was not statistically significant $(p=0.15)$. A poor outcome (score 1, 2, or 3 in GOS) was seen in $56 \%$ of the subjects, with the odds being higher in children with RSE than in children with SE (OR, 6.0; 95\% Cl, 1.6-22.3; $p=0.005)$, as were the odds of being ventilated/intubated $(\mathrm{OR}, 9.67 ; 95 \% \mathrm{Cl}, 1.03-90.4 ; p=$ 0.047 ) (Table 2). Duration of seizure at presentation was not related to either the mortality (mean \pm standard deviation [SD] duration:

Table 1. Baseline characteristics of children with SE $(n=50)$

\begin{tabular}{lcc}
\hline Characteristic & SE $(n=30)$ & RSE $(n=20)$ \\
\hline Age (years) & $5.5(3$ months to 12 years $)$ & $2.5(3$ months to 12 years $)$ \\
Seizure duration before treatment initiation $(\mathrm{n}=46)$ (minutes) & $30(30-45)$ & $35(30-45)$ \\
Fever & $20(67)$ & $14(70)$ \\
Pre-existing epilepsy & $8(27)$ & $4(20)$ \\
Developmental delay & $5(16)$ & $4(20)$ \\
Microcephaly & $4(13)$ & $4(20)$ \\
Generalized tonic clonic seizures & $29(97)$ & $17(85)$ \\
Shock at admission & $10(33)$ & $5(25)$ \\
\hline
\end{tabular}

Values are presented as median (range) or number (\%).

SE, status epilepticus; RSE, refractory status epilepticus

Table 2. Outcome* of children with SE

\begin{tabular}{llcc}
\hline Score & \multicolumn{1}{c}{ Description } & SE $(\mathrm{n}=30)$ & RSE $(\mathrm{n}=20)$ \\
\hline 1 & Death & $9(30)$ & $10(50)$ \\
2 & Persistent vegetative state & $1(3)$ & $1(5)$ \\
3 & Severe disability & $2(7)$ & $5(25)$ \\
4 & Moderate disability & $9(30)$ & $2(10)$ \\
5 & Good recovery & $9(30)$ & $2(10)$ \\
\hline
\end{tabular}

Values are presented as number (\%).

$\mathrm{SE}$, status epilepticus; RSE, refractory status epilepticus.

*Using the Glasgow Outcome Scale; $p=0.005$ for poor outcome (score 1, 2 or 3) among those with and without refractory SE. 
$35.3 \pm 5.64$ minutes vs. $34.8 \pm 5.08$ minutes; $p=0.78$ ) or the outcome (mean \pm SD duration: $35.9 \pm 5.62$ minutes vs. $34.1 \pm 5.03$ minutes; $p=0.2)$.

\section{Discussion}

In this hospital-based observational study of 50 consecutively-enrolled children with convulsive SE (including 20 with refractory convulsive SE) at a tertiary-care public hospital, we found that the majority of children showed new-onset SE. CNS infections were the most common etiology (54\%) in both groups. A poor outcome was seen in $56 \%$ of the patients, and was significantly more common in those with RSE. The duration of seizure prior to presentation was not related to progression to RSE, or to a poor outcome.

The proportion of generalized seizures varies from $63-96 \%$ in previous pediatric SE/RSE studies, ${ }^{10,15-17}$ similar to our findings (85\%). Around one-fourth of the study subjects in this study had a previous diagnosis of epilepsy, similar to recent studies on RSE from this region (16-29\%). ${ }^{8,15-17}$ These data contrast with western pediatric RSE data, where rates of $47-50 \%$ are reported, ${ }^{7}$ possibly because of a higher proportion of SE in developing countries due to neurological infections. ${ }^{8,9}$

We were able to carry out EEG in only $66 \%$ of the patients, similar to previous experiences in both pediatric ${ }^{10}$ and adult ${ }^{8,9}$ Indians. Neuroimaging was only possible in $72 \%$ of patients, with $44 \%$ having a normal scan, similar to $36 \%$ reported by Sinha et al..$^{8}$ The majority of studies from developing countries report CNS infections as the most common etiology of SE. ${ }^{8,9,16-18}$ Among pediatric studies, ${ }^{10}$ CNS infections are also the most common RSE etiology. On the other hand, in developed countries, the reported proportion of CNS infections as an etiology for RSE is low. ${ }^{6}$ Non-compliance with AEDs was an important precipitating cause of RSE in this study (13\%), similar to previous reports on adults from this region (20-27\%). ${ }^{9,19}$

A meta-analysis of pediatric RSE in $1999^{20}$ reported a mortality rate of $16 \%$; more recent studies report rates of up to $3.7 \% .{ }^{7,15}$ Adult studies from neurological tertiary-care centers in India report mortality rates of 5-35\% in RSE, 8,9 whereas pediatric Indian RSE had a mortality rate of $\sim 20 \%$ in two studies from the ICU of a tertiary-care center. ${ }^{16,17}$ We reported a higher mortality, which could be due to the smaller sample size, the decreased availability of intensive-care beds, or the more common acute CNS etiology. ${ }^{1}$ Among those discharged from the hospital, one-third were without sequelae in this study, sim- ilar to previous reports of pediatric RSE. ${ }^{6,15-17}$ However, around 50\% of pediatric RSE patients in the meta-analysis by Gilbert et al. ${ }^{20}$ had a new neurological morbidity. Poorer outcome for RSE as compared to SE has also been reported previously. ${ }^{10,21,22}$ However, similar to a previous study from Korea, ${ }^{15}$ we did not find these differences. A higher frequency of death has recently been reported in RSE patients receiving the first dose of benzodiazepine 10 minutes after seizure onset. ${ }^{23}$ We could not assess this, as none of our patients had received the first medication within 30 minutes of onset, similar to reports of prolonged seizures prior to presentation in other Indian studies. ${ }^{19}$

In addition to the small sample size, one of the major lacunae of the study was the non-availability of neuroimaging and/or EEG data for some patients. This was either due to patient death, or to the family leaving the hospital against medical advice. We could only do short-duration EEG after SE control, and could only identify one NCSE patient. In contrast, De Lorenzo et al. ${ }^{24}$ reported 14\% NCSE after SE, diagnosed on the basis of continuous EEG monitoring. The absence of ICU beds for all patients may have had an impact on the outcome, and prevents generalizing to a setting where SE is treated in the ICU. The major strength of this study was the consecutive enrollment of patients, and uniform, protocol-based management in all patients. This is the first study from a developing country that reports prospectively-controlled data on the evolution of pediatric RSE, using the newer definition of RSE. ${ }^{4}$

The high proportion of RSE patients with CNS infection as an etiology, the high mortality in the RSE group, and the poor outcome with respect to the presence of disability in the majority of survivors have important practice and policy implications. Early identification and management of SE and RSE in ICU settings could possibly reduce the mortality in this group. Given that the majority of SE and RSE patients had a CNS infection as etiology, the possibility of SE should be kept in mind and managed emergently in children with CNS infections. Other than neurological infections, the main etiology of SE/RSE in children with pre-existing epilepsy was non-compliance with AEDs; a situation that could be addressed by good patient counselling on treatment adherence. Further research may focus on prospectively identifying risk factors for SE to RSE progression in a larger patient group, and on following these patients for a longer period of time to define long-term outcomes with respect to disability, long-term seizure control, and neurodevelopmental outcomes.

In this hospital-based study of consecutively-enrolled children with convulsive SE (including 20 with RSE) at a public hospital, we found CNS infections to be the most common etiology and a poor 
outcome, especially in those with RSE. During the management of children with CNS infections, the treating physicians need to be aware of the high risk for developing RSE, and need to emergently manage this risk in an intensive-care setting, so as to reduce the mortality and morbidity from this severe neurological condition.

\section{References}

1. Neville BG, Chin RF, Scott RC. Childhood convulsive status epilepticus: epidemiology, management and outcome. Acta Neurol Scand 2007;186: 21-4.

2. Pujar SS, Martinos MM, Cortina-Borja M, et al. Long-term prognosis after childhood convulsive status epilepticus: a prospective cohort study. Lancet Child Adolesc Health 2018;2:103-11.

3. Sánchez Fernández I, Abend NS, Agadi S, et al. Gaps and opportunities in refractory status epilepticus research in children: a multi-center approach by the Pediatric Status Epilepticus Research Group (pSERG). Seizure 2014;23:87-97.

4. Owens J. Medical management of refractory status epilepticus. Semin Pediatr Neurol 2010;17:176-81.

5. Poblete R, Sung G. Status epilepticus and beyond: a clinical review of status epilepticus and an update on current management strategies in super-refractory status epilepticus. Korean J Crit Care Med 2017;32: 89-105.

6. Sahin M, Menache CC, Holmes GL, Riviello JJ. Outcome of severe refractory status epilepticus in children. Epilepsia 2001;42:1461-7.

7. Sánchez Fernández I, Jackson MC, Abend NS, et al. Refractory status epilepticus in children with and without prior epilepsy or status epilepticus. Neurology 2017;88:386-94.

8. Sinha S, Prashantha DK, Thennarasu K, Umamaheshwara Rao GS, Satishchandra P. Refractory status epilepticus: a developing country perspective. J Neurol Sci 2010;290:60-5.

9. Tripathi M, Vibha $D$, Choudhary $N$, et al. Management of refractory status epilepticus at a tertiary care centre in a developing country. Seizure 2010;19:109-11.

10. Gulati S, Kalra V, Sridhar MR. Status epilepticus in Indian children in a tertiary care center. Indian J Pediatr 2005;72:105-8.

11. Guidelines for epidemiologic studies on epilepsy. Commission on Epidemiology and Prognosis, International League Against Epilepsy. Epilepsia 1993:34:592-6.

12. Jennett $B$, Bond $M$. Assessment of outcome after severe brain damage. Lancet 1975;1:480-4.

13. Oh HH, Kwon SH. Clinical features of children with febrile status epilepticus. J Korean Child Neurol Soc 2006;14:105-12.

14. Mishra D, Sharma S, Sankhyan N, et al. Consensus guidelines of management of childhood convulsive status epilepticus. Indian Pediatr 2014;51: 975-90.

15. Kang DC, Lee YM, Lee J, Kim HD, Coe C. Prognostic factors of status epilepticus in children. Yonsei Med J 2005;46:27-33.

16. Singhi $S$, Banerjee $S$, Singhi P. Refractory status epilepticus: role of continuous diazepam infusion. J Child Neurol 1998;13:23-6.

17. Singhi $S$, Murthi A, Singhi $P$, Jayashree M. Continuous midazolam versus diazepam infusion for refractory convulsive status epilepticus. J Child Neurol 2002;17:106-10.

18. Amare A, Zenebe G, Hammack J, Davey G. Status epilepticus: clinical presentation, cause, outcome, and predictors of death in 119 Ethiopian patients. Epilepsia 2008;49:600-7.

19. Murthy JM, Jayalaxmi SS, Kanikannan MA. Convulsive status epilepticus: clinical profile in a developing country. Epilepsia 2007;48:2217-23.

20. Gilbert DL, Gartside PS, Glauser TA. Efficacy and mortality in treatment of refractory generalized convulsive status epilepticus in children: a meta-analysis. J Child Neurol 1999;14:602-9.

21. Mayer SA, Classen J, Lokin J, Mendelson F, Dennis L, Fitzsimmons BF. Refractory status epilepticus; frequency, risk factors, and impact on outcome. Arch Neurol 2002;59:205-10.

22. Towne AR, Pellock JM, Ko D, Delorenzo RJ. Determinants of mortality in status epilpticus. Epilepsia 1994;35:27-34.

23. Gaínza-Lein $M$, Sánchez Fernández $I$, Jackson $M$, et al. Association of time to treatment with short-term outcomes for pediatric patients with refractory convulsive status epilepticus. JAMA Neuro/2018;75:410-18.

24. De Lorenzo RJ, Waterhouse EJ, Towne AR, et al. Persistent non-convulsive status epilepticus after the control of convulsive status epilepticus. Epilepsia 1998;39:833-40. 